\title{
THE NASA-UC ETA-EARTH PROGRAM. I. A SUPER-EARTH ORBITING HD 7924*
}

\author{
Andrew W. Howard ${ }^{1,2}$, John Asher Johnson ${ }^{3,9}$, Geoffrey W. Marcy ${ }^{1}$, Debra A. Fischer ${ }^{4}$, Jason T. Wright ${ }^{5}$, Gregory \\ W. Henry ${ }^{6}$, Matthew J. Giguere ${ }^{4}$, Howard IsaACson ${ }^{4}$, JefF A. Valenti ${ }^{7}$, Jay Anderson ${ }^{7}$, and Nikolai E. Piskunov ${ }^{8}$ \\ ${ }^{1}$ Department of Astronomy, University of California, Berkeley, CA 94720-3411, USA \\ 2 Townes Fellow, Space Sciences Laboratory, University of California, Berkeley, CA 94720-7450, USA; howard@ astro.berkeley.edu \\ ${ }^{3}$ Institute for Astronomy, University of Hawaii, Honolulu, HI 96822, USA \\ ${ }^{4}$ Department of Physics and Astronomy, San Francisco State University, San Francisco, CA 94132, USA \\ ${ }_{5}^{5}$ Department of Astronomy, Cornell University, Ithaca, NY 14853, USA \\ ${ }^{6}$ Center of Excellence in Information Systems, Tennessee State University, 3500 John A. Merritt Blvd., Box 9501, Nashville, TN 37209, USA \\ ${ }^{7}$ Space Telescope Science Institute, 3700 San Martin Dr., Baltimore, MD 21218, USA \\ ${ }^{8}$ Department of Astronomy and Space Physics, Uppsala University, Box 515, 75120 Uppsala, Sweden \\ Received 2008 October 31; accepted 2009 January 29; published 2009 April 13
}

\section{ABSTRACT}

We report the discovery of the first low-mass planet to emerge from the NASA-UC Eta-Earth Program, a super-Earth orbiting the K0 dwarf HD 7924. Keplerian modeling of precise Doppler radial velocities reveals a planet with minimum mass $M_{P} \sin i=9.26 M_{\oplus}$ in a $P=5.398$ d orbit. Based on Keck-HIRES measurements from 2001 to 2008, the planet is robustly detected with an estimated false alarm probability of less than 0.001 . Photometric observations using the Automated Photometric Telescopes at Fairborn Observatory show that HD 7924 is photometrically constant over the radial velocity period to $0.19 \mathrm{mmag}$, supporting the existence of the planetary companion. No transits were detected down to a photometric limit of $\sim 0.5 \mathrm{mmag}$, eliminating transiting planets with a variety of compositions. HD 7924b is one of only eight planets detected by the radial velocity technique with $M_{P} \sin i<10 M_{\oplus}$ and as such is a member of an emerging family of low-mass planets that together constrain theories of planet formation.

Key words: planetary systems - stars: individual (HD 7924) - techniques: radial velocities

\section{INTRODUCTION}

The NASA-UC Eta-Earth Survey by the California Planet Search (CPS) group is a systematic search for low-mass planets $\left(\sim 3-30 M_{\oplus}\right)$ orbiting the nearest 230 GKM stars suitable for high-precision Doppler observations at the Keck Observatory. In order to place statistically significant constraints on the fraction of stars with Earth-mass planets in the habitable zone, $\eta_{\oplus}$, and theories of planet formation, each star is observed a minimum of 20 times at $\sim 1 \mathrm{~m} \mathrm{~s}^{-1}$ precision, including at least one set of high-cadence observations on consecutive nights. Planet candidates are followed up with additional high-cadence observations to confirm the candidate signal and to accurately measure the planet's orbital parameters. While Doppler surveys cannot detect $1 M_{\oplus}$ planets in the habitable zone, we will estimate $\eta_{\oplus}$ by modest extrapolation from the distribution of detected super-Earths and Neptune-mass planets in shorterperiod orbits. This paper is the first in a series to describe the planets and planetary systems emerging from the Eta-Earth Program.

The statistics of planet occurrence from the Eta-Earth Survey will offer important constraints on competing theories of planet formation (Ida \& Lin 2004; Kenyon \& Bromley 2006; Alibert et al. 2005; Mordasini et al. 2008; Ida \& Lin 2008). These models differ in assumptions about the growth rate of dust into planetesimals, the viscosity of the disk, the location and effects of the snow line in the disk, the efficacy of inward migration, the accretion of gas and water, and the relevance of planetplanet interactions. They are consistent with detections and measurements of jovian gas giants (e.g., Marcy et al. 2005a; Udry et al. 2003). However, these theories predict that planets

\footnotetext{
* Based on observations obtained at the W. M. Keck Observatory, which is operated jointly by the University of California and the California Institute of Technology. Keck time has been granted by both NASA and the University of California.

9 NSF Postdoctoral Fellow.
}

of mass $1-30 M_{\oplus}$ are rare within $1 \mathrm{AU}$, forming a "planet desert" of super-Earths $\left(M_{\mathrm{pl}} \sin i \leqslant 10 M_{\oplus}\right)$ and Neptunemass planets. All models predict that Type I migration quickly ( $\tau \sim 10^{5} \mathrm{yr}$ ) causes the rocky planets to spiral inward, destined to be lost in the star. ${ }^{10}$ Meanwhile, the more massive rocky cores $\left(M>1 M_{\oplus}\right)$ accrete gas quickly, becoming ice- or gas-giants. The resulting distribution of planetary systems is depleted of planets in the mass range $1-30 M_{\oplus}$ within $1 \mathrm{AU}$.

If the prediction of a low-mass desert is contradicted by a statistically well-defined sample of stars (such as the Eta-Earth Survey), then planet formation theory must be significantly modified with new physics. The planet desert has indeed been challenged by Mayor et al. (2009) who estimate that $30 \% \pm 10 \%$ of GK dwarfs have rocky or Neptune-mass planets inward of $50 \mathrm{~d}$ orbits. This claim is based in part on detections by the Swiss group of three Neptune-size planets orbiting HD 69830 (Lovis et al. 2006), two of three planets orbiting GJ 581 in or near the Habitable Zone (Bonfils et al. 2005; Udry et al. 2007), and a triple super-Earth system around HD 40307 (Mayor et al. 2009).

It is against this backdrop of recent discoveries and competing claims of the abundance of rocky planets that we announce the detection of a super-Earth orbiting the K dwarf HD 7924. Section 2 of this paper describes the host star, HD 7924. The spectroscopic observations and their Doppler reduction are described in Section 3. Our detection of HD 7924b, including Keplerian fitting and false alarm probability estimation, is described in Section 4. In Section 5, we consider and ultimately reject a second planetary companion based on the current data. Our photometric observations and their constraints on planetary transits are described in Section 6. We conclude in Section 7

\footnotetext{
${ }_{10}$ While some of these models (e.g., Ida \& Lin 2008) show a pile-up of low-mass planets in short-period orbits (analogous to hot Jupiters), this concentration may be artificial since the physical mechanism for stopping the migration remains uncertain.
} 
Table 1

Stellar Properties of HD 7924

\begin{tabular}{lc}
\hline \hline Parameter & Value \\
\hline Spectral type & $\mathrm{K} 0 \mathrm{~V}$ \\
$M_{V}$ & 6.056 \\
$B-V$ & 0.826 \\
$V$ & 7.185 \\
Distance $(\mathrm{pc})$ & $16.8 \pm 0.1$ \\
{$[\mathrm{Fe} / \mathrm{H}]$} & $-0.15 \pm 0.03$ \\
$T_{\text {eff }}(\mathrm{K})$ & $5177 \pm 44$ \\
$v \sin i\left(\mathrm{~km} \mathrm{~s}^{-1}\right)$ & $1.35 \pm 0.5$ \\
$\log g$ & $4.59_{-0.03}^{+0.02}$ \\
$L_{\star}\left(L_{\odot}\right)$ & $0.368_{-0.018}^{+0.019}$ \\
$M_{\star}\left(M_{\odot}\right)$ & $0.832_{-0.036}^{+0.022}$ \\
$R_{\star}\left(R_{\odot}\right)$ & $0.78 \pm 0.02$ \\
$S_{\mathrm{HK}}$ & 0.20 \\
$\log R_{\mathrm{HK}}^{\prime}$ & -4.89 \\
$P_{\text {rot }}($ days $)$ & $\sim 38$ \\
$\sigma_{\mathrm{phot}}(\mathrm{mag})$ & $<0.0017$ \\
\hline
\end{tabular}

with a discussion of HD 7924b and the emerging family of super-Earth planets.

\section{PROPERTIES OF HD 7924}

HD 7924 (HIP 6379, GJ 56.5) is a K0 dwarf whose properties are summarized in Table 1 . It is nearby, $d=16.8 \mathrm{pc}$, and relatively bright, $V=7.185$ (Perryman et al. 1997; van Leeuwen 2007). With $M_{V}=6.056$ and $B-V=0.826$, HD 7924 star lies 0.27 mag below the Hipparcos average main sequence as defined by Wright (2005).

Using the Spectroscopy Made Easy (SME) LTE spectral synthesis code, Valenti \& Fischer (2005) analyzed a highresolution, iodine-free Keck-HIRES spectrum of HD 7924 and found the effective temperature $T_{\text {eff }}=5177 \pm 30 \mathrm{~K}$, the surface gravity $\log g=4.58 \pm 0.08$, the projected rotational velocity $v \sin i=1.35 \pm 0.5 \mathrm{~km} \mathrm{~s}^{-1}$, and the iron abundance ratio $[\mathrm{Fe} / \mathrm{H}]=-0.15 \pm 0.03$ (i.e. slightly metal poor, consistent with its location below the average main sequence). Using these quantities and catalog values for $V$-band photometry, parallax, etc., they interpolated the Yonsei-Yale isochrones to obtain the luminosity $L_{\star}=0.368_{-0.018}^{+0.019} L_{\odot}$, radius $R_{\star}=0.754 \pm 0.013$ $R_{\odot}$, and mass $M_{\star}=0.80_{-0.03}^{+0.04} M_{\odot}$. Isochrones provide no useful constraint on the age because the star has not begun evolving off the main sequence.

Takeda et al. (2007) used the spectroscopically determined stellar parameters from Valenti \& Fischer (2005) and derived various stellar parameters by matching those parameters to theoretical stellar evolution models. Their values are largely consistent with Valenti \& Fischer (2005): $M_{\star}=0.832_{-0.036}^{+0.022} M_{\odot}$, $R_{\star}=0.78 \pm 0.02 R_{\odot}$, and $\log g=4.59_{-0.03}^{+0.02}$. We adopt the Takeda et al. values for the calculations below.

Measurements of the cores of the $\mathrm{Ca}$ II $\mathrm{H} \& \mathrm{~K}$ lines show that HD 7924 has modest chromospheric activity (Figure 1). We measured the chromospheric activity indices $S_{\mathrm{HK}}=0.20$ and $\log R_{\mathrm{HK}}^{\prime}=-4.89$ (Wright et al. 2004). The full set of $S_{\mathrm{HK}}$ measurements for all observations of HD 7924 is nearly constant in time (fractional rms of $1.2 \%$ ) and does not show a periodicity at or near $5.4 \mathrm{~d}$ (the planet's orbital period; see Section 4). We estimate $P_{\mathrm{rot}}=38 \mathrm{~d}$ and an age of $3.8 \mathrm{Gyr}$ using $R_{\mathrm{HK}}^{\prime}$ and $B-V$ calibration (Wright et al. 2004). Following Wright (2005), and based on the values of $S_{\mathrm{HK}}, M_{V}$, and $B-V$, we estimate a radial

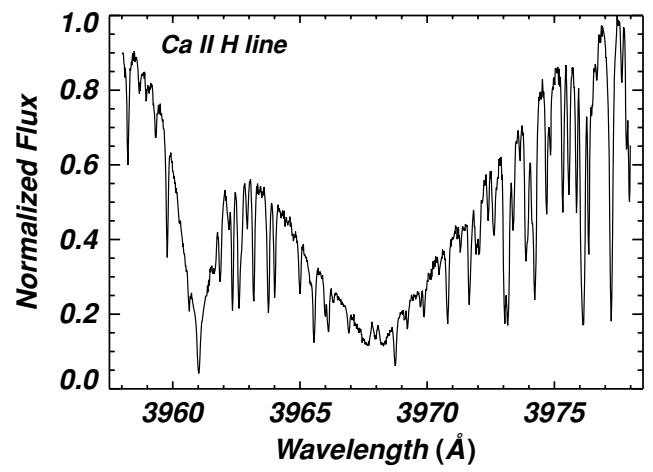

Figure 1. Ca II H line for HD 7924. Slight line core emission is seen indicating modest chromospheric activity.

velocity (RV) jitter of $2.13 \mathrm{~m} \mathrm{~s}^{-1}$. This empirical estimate for jitter accounts for RV variability due to rotational modulation of stellar surface features, stellar pulsation, undetected planets, and uncorrected systematic errors in the velocity reduction (Saar et al. 1998; Wright 2005). As explained in Section 4, jitter is added in quadrature to the RV measurement uncertainties for Keplerian fitting.

\section{OBSERVATIONS AND DOPPLER REDUCTION}

We observed HD 7924 using the HIRES echelle spectrometer (Vogt et al. 1994) on the $10 \mathrm{~m}$ Keck I telescope. The 198 observations span eight years (2001-2008) with high-cadence observations - clusters of observations on 6-12 consecutive nights-beginning in late 2005. All observations were made with an iodine cell mounted directly in front of the spectrometer entrance slit. The dense set of molecular absorption lines imprinted on the stellar spectra provides a robust wavelength fiducial against which Doppler shifts are measured, as well as strong constraints on the shape of the spectrometer instrumental profile at the time of each observation (Marcy \& Butler 1992; Valenti et al. 1995).

We measured the Doppler shift from each star-times-iodine spectrum using a modeling procedure modified from the method described by Butler et al. (1996). The most significant modification is the way we model the intrinsic stellar spectrum, which serves as a reference point for the relative Doppler shift measurements for each observation. Butler et al. used a version of the Jansson (1995) deconvolution algorithm to remove the spectrometer's instrumental profile from an iodine-free template spectrum. We instead use a new deconvolution algorithm developed by one of us (J. A. J.) that employs a more effective regularization scheme, which results in significantly less noise amplification and improved Doppler precision. We defer a more detailed description of this method to a forthcoming publication (J. A. Johnson et al. 2009, in preparation).

Figure 2 shows RV time series for four stable stars with characteristics similar to HD 7924, demonstrating our measurement precision over the past 4-11 years. In 2004 August, the Keck HIRES spectrometer was upgraded with a new detector. The previous $2 \mathrm{~K} \times 2 \mathrm{~K}$ pixel Tektronix $\mathrm{CCD}$ was replaced by an array of three $4 \mathrm{~K} \times 2 \mathrm{~K}$ pixel MIT-LL CCDs. The new detector produces significantly higher velocity precision due to its improved charge transfer efficiency and charge diffusion characteristics, smaller pixels $(15 \mu \mathrm{m}$ versus $24 \mu \mathrm{m})$, higher quantum efficiency, increased spectral coverage, and lower read noise. Our post-upgrade measurements exhibit a typical longterm rms scatter of $\sim 1.5 \mathrm{~m} \mathrm{~s}^{-1}$, compared to $\sim 2.5 \mathrm{~m} \mathrm{~s}^{-1}$ for 


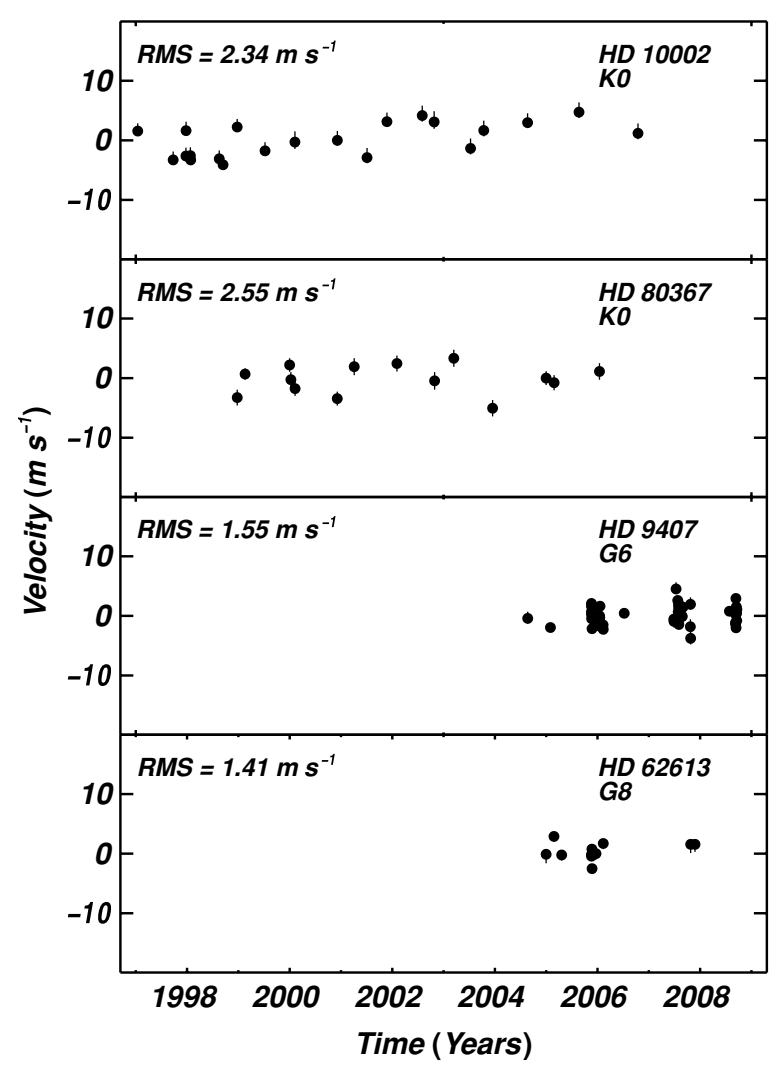

Figure 2. Radial velocity time series for four stable stars in our Keck Doppler survey that are similar to HD 7924. These stars demonstrate long-term velocity stability and improved Doppler performance for observations after the HIRES upgrade in 2004 . The bottom two panels show only post-upgrade measurements. The binned velocities with measurement uncertainties (but not jitter) are plotted. Panels are labeled with star name, spectral type, and reduced rms to a linear fit.

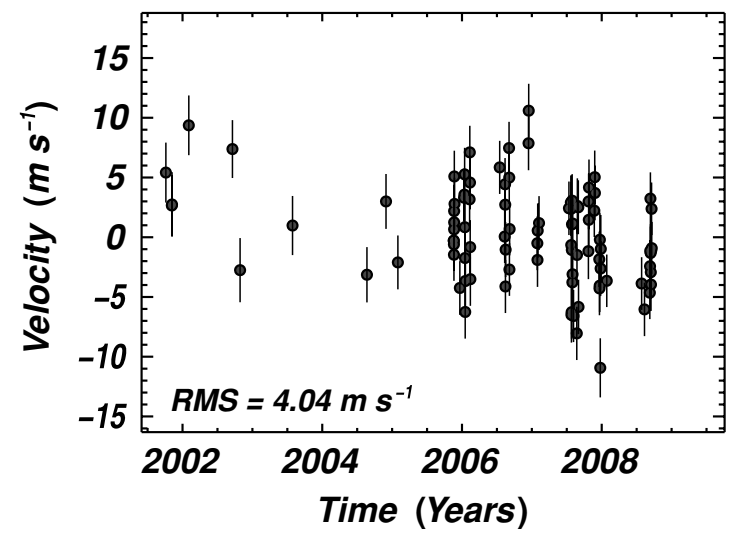

Figure 3. Time series of binned radial velocities of HD 7924 from Keck-HIRES with error bars indicating the quadrature sum of measurement uncertainties and jitter.

pre-upgrade measurements. ${ }^{11}$ The pre- and post-upgrade measurements also lack a common velocity zero point, but we've fit for and corrected this offset to within $\sim 2 \mathrm{~m} \mathrm{~s}^{-1}$ for every star observed at Keck using a large set of stable stars with many pre- and post-upgrade observations. To further limit the impact of the velocity discontinuity for this star, we let the offset float in the Keplerian fits below, effectively treating pre-upgrade and post-upgrade observations as coming from two different telescopes.

11 Measurements prior to JD 2,453,237 are pre-upgrade.

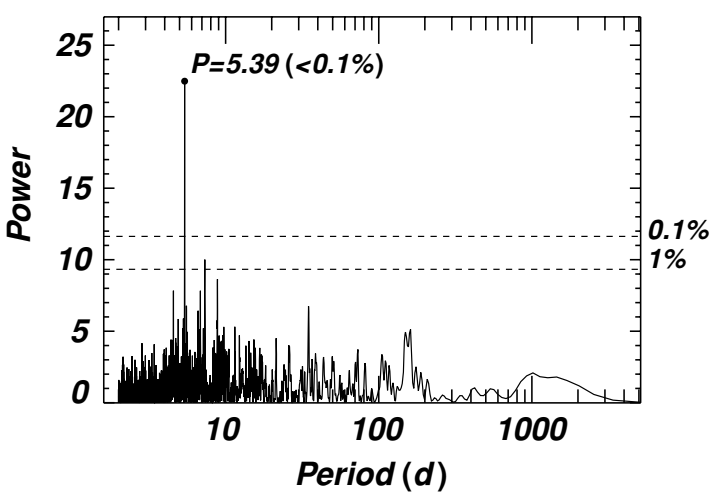

Figure 4. Lomb-Scargle periodogram of binned RV measurements of HD 7924. The tall peak near $P=5.39 \mathrm{~d}$ suggests a planet with that orbital period.

The velocities derived from the 198 observations have a median measurement uncertainty of $0.9 \mathrm{~m} \mathrm{~s}^{-1}$ for single measurements. This uncertainty is the weighted standard deviation of the mean of the velocity measured from each of the $\sim 700$ $2 \AA$ chunks in each echelle spectrum (Butler et al. 1996). In many cases, we made consecutive observations of HD 7924 to reduce the Poisson noise from photon statistics. For the Keplerian analysis below (Section 4-5), the velocities were binned in $2 \mathrm{hr}$ intervals yielding 93 measurements with an rms of $4.04 \mathrm{~m}$ $\mathrm{s}^{-1}$ about the mean and a median measurement uncertainty of $0.7 \mathrm{~m} \mathrm{~s}^{-1}$. The binned velocities and associated measurement uncertainties are listed in Table 2 and plotted as a time series in Figure 3.

\section{ORBITAL SOLUTION}

We searched the radial velocities in Table 2 for the best-fit Keplerian orbital solution using the partially linearized, leastsquares fitting procedure described in Wright \& Howard (2009). Each velocity measurement was assigned a weight constructed from the quadrature sum of the measurement uncertainty (listed in Table 2) and a stellar jitter term $\left(2.13 \mathrm{~m} \mathrm{~s}^{-1}\right)$.

The Lomb-Scargle periodogram of the velocities (Figure 4) shows a strong periodic signal near $5.39 \mathrm{~d}$. We seeded the Keplerian search with this period, and a wide variety of other trial periods, and found the best-fit orbital solution shown in Figure 5. This minimum in $\chi_{v}=\sqrt{\chi_{v}^{2}}$ implies a planet in a $5.3978 \pm 0.0015 \mathrm{~d}$ orbit around HD 7924 with a Doppler semiamplitude of $3.87 \pm 0.72 \mathrm{~m} \mathrm{~s}^{-1}$ and an orbital eccentricity of $0.17 \pm 0.16$. Note that the orbit is consistent with circular, consistent with the well-known bias against measuring $e=0$ in low amplitude systems (e.g., O'Toole et al. 2009). The bestfit orbit includes a linear trend, $d v / d t=-1.07 \pm 0.35 \mathrm{~m} \mathrm{~s}^{-1}$ $\mathrm{yr}^{-1}$. The velocity offset between pre- and post-upgrade HIRES observations was solved for and is incorporated in Table 2. Using our adopted stellar mass of $0.832 M_{\odot}$, we find a minimum planet mass of $M_{P} \sin i=9.26 M_{\oplus}$ and an orbital semimajor axis of $a=0.057 \mathrm{AU}$. The parameters of this orbital solution, and associated statistics, are listed in Table 3.

The Keplerian parameter uncertainties were derived using a Monte Carlo method (Marcy et al. 2005b) and do not account for correlations between parameter errors. With seven years of observations of a $5 \mathrm{~d}$ planet, the error on $P$ is quite small (one part in 3600) and largely uncorrelated with the errors on other Keplerian parameters. But because $K$ is relatively small, the data are nearly compatible with a family of Keplerians ranging from circular to slightly eccentric, leading to fractionally higher, 
Table 2

Radial Velocities for HD 7924

\begin{tabular}{|c|c|c|}
\hline JD-2440000 & $\begin{array}{l}\text { Radial Velocity } \\
\qquad\left(\mathrm{m} \mathrm{s}^{-1}\right)\end{array}$ & $\begin{array}{l}\text { Uncertainty } \\
\left(\mathrm{m} \mathrm{s}^{-1}\right)\end{array}$ \\
\hline 12187.970 & 5.62 & 1.3 \\
\hline 12218.998 & 2.86 & 1.3 \\
\hline 12220.015 & 2.95 & 1.7 \\
\hline 12307.770 & 9.57 & 1.3 \\
\hline 12535.955 & 7.59 & 1.2 \\
\hline 12575.915 & -2.55 & 1.6 \\
\hline 12850.115 & 1.19 & 1.3 \\
\hline 13239.082 & -2.93 & 0.9 \\
\hline 13338.794 & 3.20 & 0.9 \\
\hline 13400.804 & -1.90 & 0.7 \\
\hline 13692.825 & -0.08 & 0.5 \\
\hline 13693.006 & -0.09 & 0.5 \\
\hline 13693.777 & -0.40 & 0.5 \\
\hline 13693.952 & -1.26 & 0.5 \\
\hline 13694.820 & 0.87 & 0.5 \\
\hline 13694.956 & 2.40 & 0.5 \\
\hline 13695.010 & 1.45 & 0.6 \\
\hline 13695.898 & 5.29 & 0.3 \\
\hline 13696.929 & 2.99 & 0.5 \\
\hline 13724.850 & -4.04 & 0.7 \\
\hline 13746.799 & 3.51 & 0.7 \\
\hline 13748.790 & 3.77 & 0.6 \\
\hline 13749.785 & 5.47 & 0.6 \\
\hline 13750.795 & 1.05 & 0.6 \\
\hline 13751.831 & -1.53 & 0.7 \\
\hline 13752.836 & -6.05 & 0.7 \\
\hline 13753.801 & -3.46 & 0.6 \\
\hline 13775.767 & 3.38 & 0.7 \\
\hline 13776.805 & 7.31 & 0.6 \\
\hline 13777.805 & 4.78 & 0.7 \\
\hline 13778.799 & -0.62 & 0.7 \\
\hline 13779.823 & -3.30 & 0.7 \\
\hline 13933.124 & 6.05 & 0.6 \\
\hline 13959.130 & 0.26 & 0.6 \\
\hline 13961.070 & 4.64 & 0.5 \\
\hline 13962.067 & 2.93 & 0.5 \\
\hline 13963.104 & -3.91 & 0.6 \\
\hline 13964.134 & -0.83 & 0.9 \\
\hline 13982.026 & 7.67 & 0.5 \\
\hline 13983.083 & 5.20 & 0.6 \\
\hline 13983.987 & -2.50 & 0.6 \\
\hline 13985.005 & 0.89 & 0.6 \\
\hline 14083.895 & 8.07 & 0.7 \\
\hline 14084.837 & 10.80 & 0.7 \\
\hline 14129.917 & -0.30 & 0.7 \\
\hline 14130.714 & -1.69 & 0.7 \\
\hline 14131.780 & 0.76 & 0.8 \\
\hline 14138.764 & 1.39 & 0.7 \\
\hline 14295.133 & 2.63 & 0.7 \\
\hline 14305.135 & 3.12 & 0.8 \\
\hline 14306.136 & 2.58 & 0.7 \\
\hline 14307.122 & -0.45 & 0.6 \\
\hline 14308.117 & -6.29 & 0.9 \\
\hline 14309.108 & -6.06 & 0.6 \\
\hline 14310.108 & -0.86 & 0.6 \\
\hline 14311.093 & 1.29 & 0.6 \\
\hline 14312.089 & 3.27 & 0.6 \\
\hline 14313.084 & -3.57 & 0.7 \\
\hline 14314.089 & -2.89 & 0.7 \\
\hline 14315.106 & 1.35 & 0.6 \\
\hline 14319.125 & -6.39 & 0.5 \\
\hline 14336.084 & -7.84 & 0.7 \\
\hline 14337.143 & -1.27 & 1.0 \\
\hline 14339.112 & 2.83 & 0.9 \\
\hline 14344.047 & 2.72 & 0.7 \\
\hline
\end{tabular}

Table 2

(Continued)

\begin{tabular}{|c|c|c|}
\hline JD-2440000 & $\begin{array}{l}\text { Radial Velocity } \\
\left(\mathrm{m} \mathrm{s}^{-1}\right)\end{array}$ & $\begin{array}{c}\text { Uncertainty } \\
\left(\mathrm{m} \mathrm{s}^{-1}\right)\end{array}$ \\
\hline 14345.147 & -5.63 & 0.8 \\
\hline 14396.864 & -0.96 & 1.0 \\
\hline 14397.921 & 3.20 & 0.9 \\
\hline 14398.932 & 1.66 & 1.0 \\
\hline 14399.877 & 4.37 & 1.0 \\
\hline 14427.831 & 2.43 & 0.6 \\
\hline 14429.895 & 5.23 & 0.7 \\
\hline 14430.934 & 3.91 & 0.8 \\
\hline 14453.738 & -1.64 & 0.9 \\
\hline 14454.786 & -4.08 & 0.7 \\
\hline 14455.788 & -3.85 & 0.6 \\
\hline 14456.771 & 0.00 & 0.8 \\
\hline 14458.922 & -10.72 & 1.3 \\
\hline 14460.768 & -2.41 & 0.9 \\
\hline 14461.695 & -0.77 & 1.9 \\
\hline 14492.794 & -3.44 & 0.5 \\
\hline 14675.111 & -3.67 & 0.6 \\
\hline 14690.113 & -5.83 & 0.7 \\
\hline 14718.021 & -4.44 & 0.6 \\
\hline 14719.063 & -2.22 & 0.5 \\
\hline 14720.076 & -0.94 & 0.6 \\
\hline 14721.069 & 3.44 & 0.5 \\
\hline 14722.117 & -1.11 & 0.6 \\
\hline 14723.038 & -2.72 & 0.6 \\
\hline 14724.006 & -3.75 & 0.6 \\
\hline 14725.049 & -0.92 & 0.6 \\
\hline 14727.066 & 2.59 & 0.5 \\
\hline 14727.989 & -0.71 & 0.5 \\
\hline
\end{tabular}

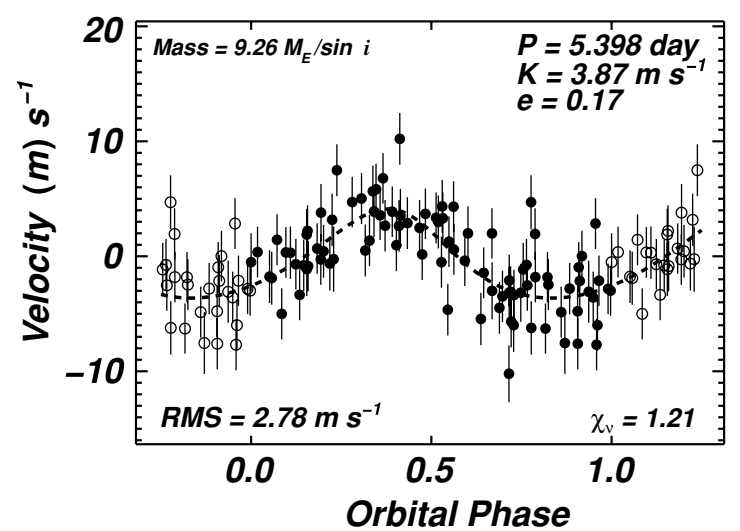

Figure 5. Single-planet Keplerian model for the radial velocities of HD 7924, as measured by Keck-HIRES. The dashed line shows the best-fit Keplerian orbital solution representing a $9.26 M_{\oplus}$ (minimum mass) planet in a $5.398 \mathrm{~d}$ orbit. Filled circles represent phased and binned radial velocities, while the open circles show the same velocities wrapped one orbital phase. The error bars show the quadrature sum of measurement uncertainties and $2.13 \mathrm{~m} \mathrm{~s}^{-1}$ jitter.

correlated errors on the other parameters (especially $e$ and $\omega$ ). Our estimate for a possible time of mid-transit (see Section 6) accounts for these correlated parameters, thereby reclaiming some of the clock-like precision of the period estimate.

As we show in Section 6, HD 7924 is photometrically stable, particularly on the short timescales near the $5.4 \mathrm{~d}$ Keplerian period. Thus, the RV signal we detected is almost certainly not due to rotational modulation of spots or other stellar surface features.

We considered the possibility that the velocity periodicity represented by the Keplerian orbital fit in Figure 5 arose from 


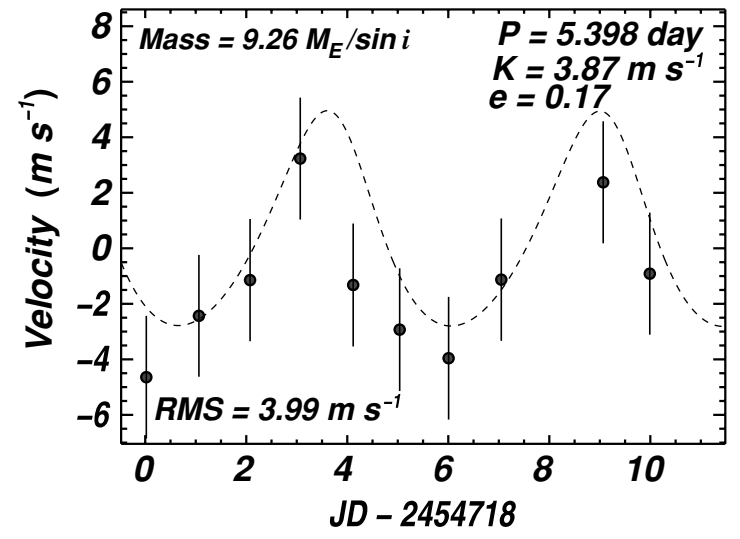

Figure 6. High-cadence radial velocities of HD 7924 during a long observing run. Measurements were made on 10 of 11 consecutive nights. The velocities show temporal coherence over two orbital phases that matches the best-fit Keplerian orbital solution to all of the velocities for HD 7924 (the same orbital solution as in Figure 5). The error bars show the quadrature sum of measurement uncertainties and $2.13 \mathrm{~m} \mathrm{~s}^{-1}$ jitter.

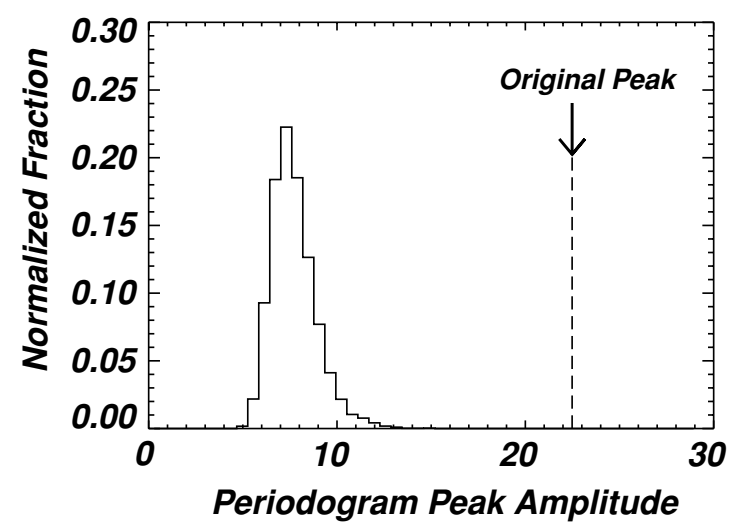

Figure 7. FAP determination for HD 7924 using periodogram peak height. The histogram shows the peak amplitudes from the periodograms of $10^{4}$ synthetic RV data sets, selected with replacement from the measured RVs. None of the trials yielded a periodogram peak amplitude greater than the original fit, implying FAP $<10^{-4}$.

chance fluctuations in the velocities. The amplitude of our orbital solution is less than $4 \mathrm{~m} \mathrm{~s}^{-1}$, which is within a factor of two of the quadrature sum of measurements uncertainties and jitter, so a careful treatment is deserved. We tested the null hypothesis in many ways, including several estimates of the false alarm probability (FAP).

First, we note that the unreduced value of $\chi^{2}$ decreased from 255 for a linear fit to the data $\left(N_{\mathrm{obs}}-2=91\right.$ degrees of freedom) to 125 for the single-planet Keplerian fit (85 degrees of freedom). This corresponds to a decrease in $\chi_{v}$ from 1.68 to 1.21 , and a decrease in the reduced velocity rms from 3.81 to $2.78 \mathrm{~m} \mathrm{~s}^{-1}$.

Figure 6 shows that all of the velocities measured during a recent 11-night observing run appear consistent in period, phase, and amplitude with the overplotted best-fit Keplerian solution that was derived from the complete set of 93 velocities.

We also computed multiple false alarm probabilities associated with the chance arrangement of random velocities masquerading as a coherent signal (Marcy et al. 2005b; Cumming 2004). Figure 7 shows the periodogram-based FAP test. In this test, we created $10^{4}$ synthetic data sets by drawing (with replacement) the velocities and associated errors from the set of measured velocities and using the actual observation times. A

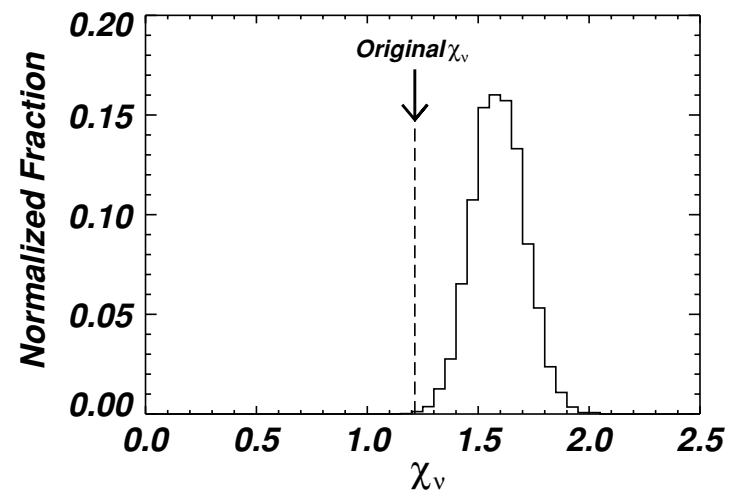

Figure 8. FAP determination for HD 7924 using $\chi_{\nu}$. The histogram shows $\chi_{v}$ from the best-fit Keplerian solution to 1000 synthetic RV data sets, selected with replacement from the measured RVs. None of the trials yielded a $\chi_{v}$ lower than the original fit, implying FAP $<0.001$.

periodogram was computed for each synthetic data set and the peak amplitudes (typically in the range $\sim 5-10$ ) are plotted as a histogram in Figure 7. This distribution is cleanly separated from the periodogram peak amplitude of the measured velocities, implying a strong coherence in the measured velocities well in excess of random fluctuations. We conclude that it is extremely unlikely (FAP $\ll 10^{-4}$ ) that a rearrangement of the measured velocities would result in a Fourier decomposition with as much power in a single mode as we observed. That is, a circular or nearly circular orbit of equal or greater amplitude would almost never be falsely detected.

While instructive, the periodogram-based test underestimates the true FAP associated with searching for a Keplerian signal because it only measures the strength of sinusoidal signals. To overcome this limitation we use a test based on $\chi_{v}$ for a full Keplerian fit, instead of periodogram peak height. As with the previous test, the synthetic data sets were constructed by drawing with replacement from the measured velocities. Figure 8 shows the distribution of $\chi_{v}$ for the synthetic data sets as well as the value of $\chi_{\nu}$ for the Keplerian fit to the original, unscrambled velocities. None of the synthetic data sets had a best-fit Keplerian with $\chi_{v}$ lower than for the unscrambled velocities, implying an FAP for this scenario of $<0.001$.

This FAP based on $\chi_{v}$ is also instructive, but is probably too conservative for at least two reasons. (Note that these caveats are irrelevant for HD 7924b since we have already shown that the FAP is very small, but are important for the possible second companion considered in Section 5 and for future planetary systems.) First, it considers all Keplerian solutions equally believable, independent of orbital eccentricity. To be sure, nature occasionally produces planetary systems with eccentricities of $\sim 0.9$ (e.g., HD 80606; Naef et al. 2001) that would have seemed unbelievable not long ago. Yet, when these models arise as solutions to synthetic data sets, the high eccentricity and low $\chi_{\nu}$ are often driven by a few aberrant points that sometimes arise in a data set created from non-Gaussian noise drawn with replacement. When such solutions are proposed for real data, they are often regarded with skepticism until the eccentric Keplerian hypothesis can be tested with additional measurements around the time of periastron passage. Thus, in accommodating all Keplerian signals, this test overestimates the FAP.

The second reason the $\chi_{v}$-based FAP is sometimes too conservative is also a failure of overaccommodation. This test (as well as the periodogram-based FAP test) assumes that the observed 


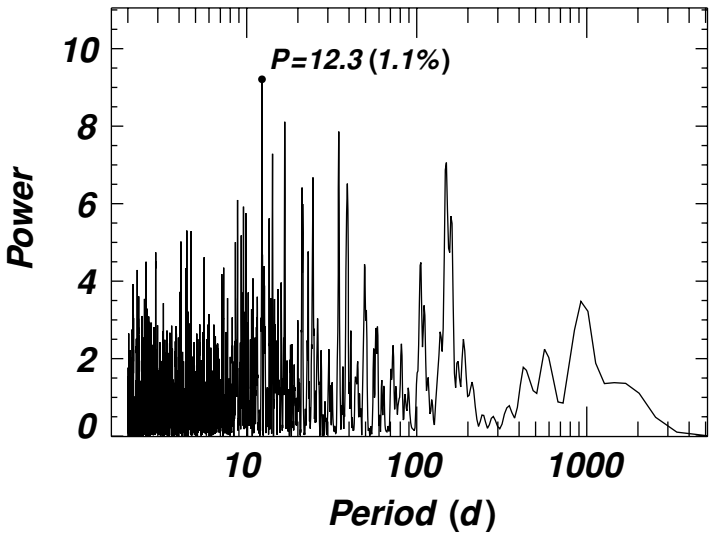

Figure 9. Lomb-Scargle periodogram of velocity residuals to the single-planet fit show in Figure 5. The peaks at 12.3, 16.7, 34.8, and 147.5 d suggest a possible second planet at one of these periods. Additional measurements are needed to tease out such a weak signal, if present.

velocity variation is completely due to noise. However, we have independent estimates of the expected velocity variability of a given star from the Doppler analysis pipeline, activity indices, and comparison stars. For HD 7924, the measurement uncertainties $\left(0.7 \mathrm{~m} \mathrm{~s}^{-1}\right)$ and jitter $\left(2.13 \mathrm{~m} \mathrm{~s}^{-1}\right)$ predict the level of velocity $\mathrm{rms}\left(2.3 \mathrm{~m} \mathrm{~s}^{-1}\right)$ in the absence of a planetary companion. Thus, when interpreting this FAP, we should discount it by the probability that the observed velocity variability is due to noise.

On balance, we reject the null hypothesis and with confidence attribute the observed velocity variation to a planet orbiting HD 7924 with a period of $5.398 \mathrm{~d}$.

\section{POSSIBLE SECOND COMPANION}

Motivated by the success of our single-Keplerian fit and the higher than expected velocity rms to that fit $\left(2.78 \mathrm{~m} \mathrm{~s}^{-1}\right)$, we performed a search for a double-Keplerian fit. The LombScargle periodogram of the residuals to the single-planet fit (Figure 9) shows several short-period narrow peaks with modest power (at 12.3, 16.7, $34.8 \mathrm{~d}$ ) and an intermediate-period peak with broad power (at $147.5 \mathrm{~d}$ ). Each of these peaks represents a possible second planet in the system.

We seeded the Keplerian search with several trial two-planet solutions. The first planet was always seeded with the orbital parameters for the best-fit single-planet model in Section 4. The second planet was seeded with the largest peaks in the periodogram of single-planet residuals (Figure 9) and a wide variety of other trial periods. All of the parameters were allowed to vary during the fitting process (Wright \& Howard 2009). None of these two-planet fits changed the orbital parameters for HD 7924b substantially. The top four two-planet solutions had best-fit second-planet periods of 12.3, 34.8, 16.7, and $147.5 \mathrm{~d}$, and best-fit eccentricities of $0.72,0.76,0.44$, and 0.83 , respectively. The high eccentricities raise our suspicion that the signals may be due to noise and increase the need for a robust tests of the null hypothesis.

We calculated FAPs for each of these two-planet models, building on the methods described in Section 4. Synthetic data sets were constructed by drawing with replacement from the residuals to the best-fit single-planet solution and adding the coherent, best-fit solution back to the scrambled residuals. We performed a thorough search for the best-fit two-Keplerian model (as above) on each synthetic data set. False alarms were triggered when a synthetic data set had a lower value of $\chi_{\nu}$ than the original, unscrambled data. All of the two-planet models had an FAP $>20 \%$, even when we restricted the scrambled trials to low-eccentricity solutions. Thus, we do not consider any of these signals to be viable planet candidates at the present time. Nevertheless, we will continue to hunt for additional planets orbiting HD 7924 by making additional observations and by refining our Doppler analysis algorithms to reanalyze our extant Doppler spectra more precisely.

\section{PHOTOMETRIC OBSERVATIONS}

We acquired photometric observations of HD 7924 with the T12 $0.8 \mathrm{~m}$ automated photometric telescope (APT) at Fairborn observatory in southern Arizona. Our brightness measurements were made between 2006 December and 2008 October and cover the last part of the 2006-07, the complete 2007-08, and the first part of the 2008-09 observing seasons. The T12 APT and its precision photometer are very similar to the T8 APT described in Henry (1999). The precision photometer uses two temperature-stabilized EMI 9124QB photomultiplier tubes to measure photon count rates simultaneously through Strömgren $b$ and $y$ filters.

The telescope was programmed to gather observations of HD 7924 with respect to three nearby comparison stars in the following sequence: DARK, A, B, C, D, A, SKY $\mathrm{A}, \mathrm{B}, \mathrm{SKY}_{\mathrm{B}}$, C, $\mathrm{SKY}_{\mathrm{C}}, \mathrm{D}, \mathrm{SKY}_{\mathrm{D}}, \mathrm{A}, \mathrm{B}, \mathrm{C}, \mathrm{D}$. The comparison stars A, $\mathrm{B}$, and $\mathrm{C}$ are HD $4295(V=6.39, B-V=0.42, \mathrm{~F} 3 \mathrm{~V})$, HD $10971(V=6.94, B-V=0.49, \mathrm{~F} 5)$, and HD 10259 $(V=6.59, B-V=1.04, \mathrm{G} 5)$, respectively, while star D is HD $7924(V=7.17, B-V=0.83, \mathrm{~K} 0)$.

Each complete sequence, referred to as a group observation, was reduced to form three independent measures of each of the six differential magnitudes $\mathrm{D}-\mathrm{A}, \mathrm{D}-\mathrm{B}, \mathrm{D}-\mathrm{C}, \mathrm{C}-\mathrm{A}, \mathrm{C}-\mathrm{B}$, and $\mathrm{B}-\mathrm{A}$. The differential magnitudes were corrected for differential extinction with nightly extinction coefficients and transformed to the standard Strömgren system with yearly mean transformation coefficients. The three independent measures of each differential magnitude were combined, giving one mean data point per complete sequence for each of the 6 differential magnitudes. To filter any observations taken under nonphotometric conditions, an entire group observation was discarded if the standard deviation of any of the six mean differential magnitudes exceeded $0.01 \mathrm{mag}$. Finally, we combined the Strömgren $b$ and $y$ differential magnitudes into a single $(b+y) / 2$ passband to improve the precision.

Our complete data set consists of 192, 435, and 145 good group observations from the 2006-07, 2007-08, and 2008-09 observing seasons, respectively, for a total 772 . To minimize the effect of any low-level intrinsic variation in the three comparison stars, we averaged the three $\mathrm{D}-\mathrm{A}, \mathrm{D}-\mathrm{B}$, and $\mathrm{D}-\mathrm{C}$ differential magnitudes of HD 7924 within each group into a single value, representing the difference in brightness between HD 7924 and the mean of the three comparison stars: $D-(A+B+C) / 3$. The standard deviations of these ensemble differential magnitudes for each of the three observing seasons are 1.77, 1.85, and 1.47 mmag, respectively. The standard deviation of the complete data set is $1.76 \mathrm{mmag}$. These values are comparable to the typical precision of a single observation with our $0.8 \mathrm{~m}$ APTs (Henry 1999), indicating there is little or no photometric variability in HD 7924 or in the three comparison stars. Solar-type stars often exhibit brightness variations caused by cool, dark photospheric spots as they are carried into and out of view by stellar rotation (e.g., Gaidos et al. 2000). However, periodogram analysis of the 

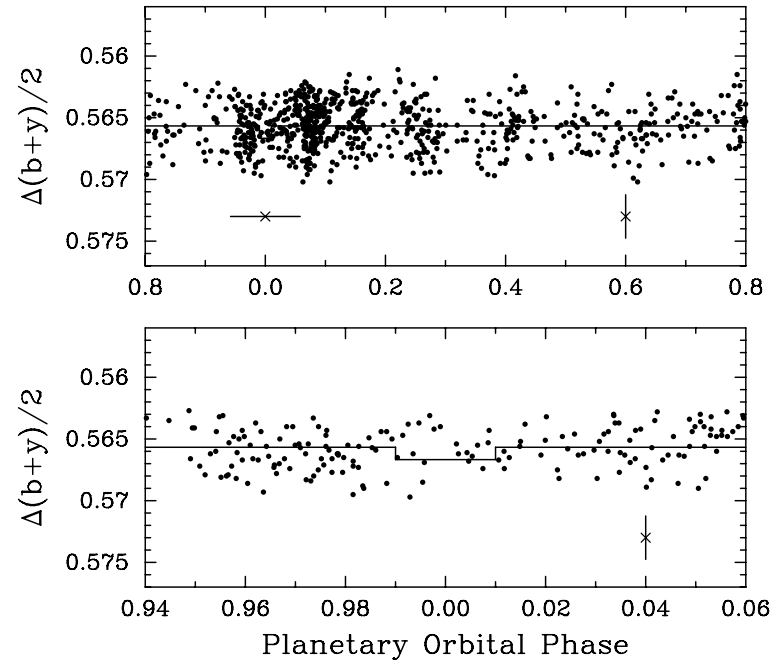

Figure 10. Top Panel: the 772 ensemble $D-(A+B+C) / 3$ photometric observations of HD 7924 in the combined Strömgren $(b+y) / 2$ passband, acquired with the T12 $0.8 \mathrm{~m}$ APT over three observing seasons and plotted modulo the $5.3978 \mathrm{~d}$ orbital period of the super-Earth companion. Phase 0.0 corresponds to the predicted time of mid-transit. A least-squares sinusoidal fit at the orbital period yields a semi-amplitude of only $0.19 \pm 0.09 \mathrm{mmag}$. Bottom Panel: the photometric observations of HD 7924 near the predicted time of transit replotted with an expanded scale on the abscissa. The solid curve shows the predicted time of transit with a drop in stellar brightness of $1 \mathrm{mmag}$. The error bar in the lower right of both panels represents the mean precision of a single observation $(1.7 \mathrm{mmag})$. The error bar immediately below the transit in the top panel represents the uncertainty in the predicted time of mid-transit ( \pm 0.06 phase units). The width of the plot in the bottom panel corresponds to this uncertainty in the transit timing.

individual three seasons of HD 7924 and of the three observing seasons taken together, confirms the lack of any photometric periodicity between $1 \mathrm{~d}$ and $100 \mathrm{~d}$. This is consistent with the star's modest level of chromospheric activity and its low $v \sin i$.

The 772 ensemble $(b+y) / 2$ differential magnitudes of HD 7924 are plotted in the top panel of Figure 10. Phases are computed from the Keplerian orbital period $P=5.3978$ $\pm 0.0015 \mathrm{~d}$ (Table 3 ) and the epoch JD 2,454,727.99 \pm 0.16 , a recent time of mid-transit derived from the orbital elements. The standard deviation of a single observation from the mean of the entire data set is 1.76 mmag. A least-squares sinusoidal fit on the orbital period yields a semi-amplitude of only $0.19 \pm 0.09$ mmag. This very low limit to photometric variability on the RV period is strong evidence that the low-amplitude RV variations observed in the star are, in fact, due to planetary reflex motion and not to activity-induced intrinsic variations in the star itself (e.g., Paulson et al. 2004).

The photometric observations of HD 7924 near the predicted time of transit are replotted with an expanded horizontal scale in the bottom panel of Figure 10. The solid curve shows the predicted time ( 0.00 phase units) and duration $( \pm 0.01$ phase units) of transits with an arbitrary drop of 1 mmag from the mean brightness level of all the observations. The error bar in the lower right of both upper and lower panels represents the mean precision of a single observation $(1.7 \mathrm{mmag})$. The horizontal error bar immediately below the transit in both panels represents the $2 \sigma$ uncertainty in the predicted time of midtransit ( $\pm 0.32 \mathrm{~d}$ or \pm 0.06 phase units). The a priori probability of HD 7924b transiting its host star is 0.06 . As discussed in Section 7, the predicted depth for a transit of HD 7924b ranges from $6 \mathrm{mmag}$ down to $0.3 \mathrm{mmag}$, depending on the composition of the planet. Transit depths of 2-3 mmag or more are easily excluded by the data. The 20 observations within
Table 3

Orbital Solution for HD 7924b

\begin{tabular}{lc}
\hline \hline Parameter & Value \\
\hline$P($ days $)$ & $5.3978 \pm 0.0015$ \\
$e$ & $0.17 \pm 0.16$ \\
$K\left(\mathrm{~m} \mathrm{~s}^{-1}\right)$ & $3.87 \pm 0.72$ \\
$T_{p}(\mathrm{JD}-2,440,000)$ & $14,727.27 \pm 0.87$ \\
$\omega(\mathrm{deg})$ & $25 \pm 60$ \\
$d v / d t\left(\mathrm{~m} \mathrm{~s}^{-1} \mathrm{yr}^{-1}\right)$ & $-1.07 \pm 0.35$ \\
$M \sin i\left(M_{\oplus}\right)$ & 9.26 \\
$a(\mathrm{AU})$ & 0.057 \\
$N_{\text {obs }}($ binned $)$ & 93 \\
Median binned uncertainty $\left(\mathrm{m} \mathrm{s}^{-1}\right)$ & 0.7 \\
Assumed jitter $\left(\mathrm{m} \mathrm{s}^{-1}\right)$ & 2.13 \\
Reduced rms to fit $\left(\mathrm{m} \mathrm{s}^{-1}\right)$ & 2.78 \\
$\sqrt{\chi_{v}^{2}}$ & 1.21 \\
FAP (periodogram peak $)$ & $<0.0001$ \\
FAP $\left(\chi_{v}\right.$ for Keplerian fit $)$ & $<0.001$ \\
\hline
\end{tabular}

the nominal transit window between phases 0.99 and 1.01 have a mean brightness just 0.06 mmag fainter than the mean light curve level. To estimate the limiting depth of detectable transits from our current data set, we segregated the observations between phases 0.93 and 0.07 into seven bins each 0.002 phase units in width. We computed the mean depth of each bin and compared them to the mean brightness level of all the observations. The differences ranged from $0.46 \mathrm{mmag}$ fainter to $1.12 \mathrm{mmag}$ brighter than the overall mean brightness. The standard deviation of the seven difference values is $0.4 \mathrm{mmag}$. Thus, we conclude that transits deeper than 2-3 mmag do not occur, but additional observations around predicted transit times will be required to improve the lower limit or confirm transit depths around $0.5 \mathrm{mmag}$ or less.

\section{DISCUSSION}

We present the detection of HD 7924b, a super-Earth planet with $M_{P} \sin i=9.26 M_{\oplus}$ in a $P=5.398 \mathrm{~d}$ orbit around a K0 dwarf. This paper is the first in a series of super-Earth $\left(M \sin i<10 M_{\oplus}\right)$ and Neptune-mass planet announcements from the NASA-UC Eta-Earth Program. The 230 stars in this survey are nearby $(d<25 \mathrm{pc})$, bright enough for $\sim 1 \mathrm{~m} \mathrm{~s}^{-1}$ RV measurements with Keck-HIRES $(V<11$; some faint stars suffer from slightly poorer Doppler precision), have low to moderate chromospheric activity $\left(\log R_{\mathrm{HK}}^{\prime}<-4.7\right)$, and are within two magnitudes of the main sequence. Several candidate super-Earth and Neptune-mass planets are emerging from the survey. Some of these require additional follow-up observations to verify the orbit and accurately estimate Keplerian parameters. Once the survey and follow-up observations are complete, the statistics of low-mass planets will offer strong constraints on planet-formation theory. Just as successful theories of planet formation must reproduce the observed planet distribution in mass-period space and other parameterizations for Jupiterand Saturn-mass planets (Cumming et al. 2008), they must do the same for low-mass planets. In this regard, HD 7924b is an interesting first detection for the Eta-Earth survey. Its minimum mass of $9 M_{\oplus}$ places it clearly in the "planet desert," while its small semimajor axis $(0.057 \mathrm{AU})$ pushes it into the pileup of short-period "hot super-Earths" and "hot Neptunes" seen in some simulations (Ida \& Lin 2008). The existence of this population depends crucially on an unknown mechanism stopping Type I migration and parking the planets in shortperiod orbits. The statistical power of the full Eta-Earth survey 
Table 4

Planetary and Stellar Parameters for RV-Detected Super-Earths $\left(M_{\mathrm{pl}} \sin i \leqslant\right.$ $\left.10 M_{\oplus}\right)$

\begin{tabular}{lcccccc}
\hline \hline Planet & $\begin{array}{c}M_{\mathrm{pl}} \sin i \\
\left(M_{\oplus}\right)\end{array}$ & $\begin{array}{c}P \\
(\mathrm{~d})\end{array}$ & $\begin{array}{c}a \\
(\mathrm{AU})\end{array}$ & $\begin{array}{c}\text { Spec. } \\
\left(M_{\odot}\right)\end{array}$ & $M_{\star}$ & {$[\mathrm{Fe} / \mathrm{H}]$} \\
\hline HD 7924b & 9.3 & 5.4 & 0.057 & $\mathrm{~K} 0 \mathrm{~V}$ & 0.83 & -0.15 \\
HD 40307b & 4.2 & 4.3 & 0.047 & $\mathrm{~K} 2.5 \mathrm{~V}$ & 0.77 & -0.31 \\
HD 40307c & 6.9 & 9.6 & 0.081 & $\mathrm{~K} 2.5 \mathrm{~V}$ & 0.77 & -0.31 \\
HD 40307d & 9.2 & 20.5 & 0.134 & K2.5 V & 0.77 & -0.31 \\
GJ 176b & 8.4 & 8.8 & 0.066 & M2 V & 0.53 & -0.1 \\
GJ 581c & 5.0 & 12.9 & 0.073 & M3 V & 0.31 & -0.33 \\
GJ 581d & 7.7 & 83.6 & 0.25 & M3 V & 0.31 & -0.33 \\
GJ 876d & 5.7 & 1.9 & 0.021 & M4 V & 0.32 & -0.12 \\
\hline
\end{tabular}

Notes. Planetary parameters (minimum mass, orbital period, and semimajor axis) of known super-Earth planets $\left(M_{\mathrm{pl}} \sin i \leqslant 10 M_{\oplus}\right)$ and stellar parameters (spectral type, mass, and iron abundance ratio) of their host stars. Only planets detected by the RV method are shown. HD $40307 \mathrm{~b} / \mathrm{c} / \mathrm{d}$ were discovered by Mayor et al. (2009), GJ 176b by Forveille et al. (2009), GJ 581c/d by Udry et al. (2007), and GJ 876d by Rivera et al. (2005).

is needed to check whether HD 7924b (as well as other superEarths listed in Table 4) is a member of this hypothesized class of planets or an outlier in a mostly barren patch of planet parameter space.

HD 7924b is one of only eight super-Earth planets detected by the RV technique and published (or submitted) in the refereed literature (Table 4). ${ }^{12}$ The other planets are: HD $40307 \mathrm{~b} / \mathrm{c} / \mathrm{d}$, the triple super-Earth system (Mayor et al. 2009); GJ 581c/d, the two planets in or near the habitable zone (Udry et al. 2007); GJ 876d, the super-Earth in the same system as two resonant, intermediate-period Jupiters (Rivera et al. 2005); and GJ 176b, an $8 M_{\oplus}$ super-Earth (Forveille et al. 2009).

Table 4 reveals some emerging properties of super-Earths, albeit with the limited statistical power of only eight detections and through the lens of possible observational selection effects. Only three of these planets have periods longer than $10 \mathrm{~d}$ and only one has a period longer than $30 \mathrm{~d}$. All of these planets are in low-eccentricity orbits, consistent with circular. As with higher-mass planets in short-period orbits, this is probably due to tidal circularization. It is curious that HD 7924b and GJ 176b are the only Doppler planets in this mass range not in known multiplanet systems. This is quite different from the $14 \%$ of higher-mass planets in known multiplanet systems (Wright et al. 2009).

The host stars of known super-Earths also show emerging trends. While Jovian-mass planets are found preferentially around higher-mass stars (J. A. Johnson et al. 2007), the preliminary statistics of super-Earths are consistent with the opposite trend. The known super-Earth host stars are all $\mathrm{K}$ and M dwarfs in the mass range $0.31-0.83 M_{\odot}$. Current Doppler surveys are capable of detecting super-Earths orbiting $\mathrm{G}$ dwarfs (with reduced sensitivity), but none have been reported in the literature as of 2008 October. Note that super-Earths around most F stars and intermediate-mass subgiants with $M_{\star} \gtrsim 1.3$ $M_{\odot}$ ("retired" A stars) are undetectable by the Doppler method due to higher intrinsic RV variability observed in these targets.

The metallicities of super-Earth host stars also show the opposite trend seen in higher-mass stars. Note that all of the detected super-Earths orbit metal-poor stars, in sharp contrast

\footnotetext{
12 Table 4 does not include super-Earth planets detected by microlensing (e.g. Bennett et al. 2009; Beaulieu et al. 2006) or by pulsar timing (Wolszczan \& Frail 1992). The microlensing planets are relevant for statistical estimates of $\eta_{\oplus}$ around main sequence stars, which is left to future work.
}

to the positive correlation observed between host star metallicity and Jupiter-mass planet occurrence (Fischer \& Valenti 2005).

Additional planets and nondetections from unbiased stellar samples are needed to determine if the above trends reflect physical processes or selection effects. While planet detectability depends on many factors including the planet's Doppler semi-amplitude $K$, the amplitude of systematic and astrophysical errors, measurements precision, the number and timing of observations, the number of planets in the system, etc., we can say roughly that the lowest-mass detectable planets have $K$ comparable to the velocity rms of long-term stable stars (see Figure 2). Since the Doppler semi-amplitude of superEarths scales as

$$
K=\frac{3.7 \mathrm{~m} \mathrm{~s}^{-1}}{\left(1-e^{2}\right)^{1 / 2}}\left(\frac{P}{5 \mathrm{~d}}\right)^{-1 / 3}\left(\frac{M_{\star}}{M_{\odot}}\right)^{-2 / 3} \frac{M_{\mathrm{pl}} \sin i}{10 M_{\oplus}},
$$

RV surveys are biased against low-mass, long-period planets orbiting high-mass stars. For comparison, the known superEarths listed in Table 4 have $K=2.0-6.5 \mathrm{~m} \mathrm{~s}^{-1}$.

HD 7924 is nearby and bright, making plausible the detection of its short-period planet by other techniques. While our photometric observations preclude a transit down to the level of approximately $0.5 \mathrm{mmag}$ during the times described in Section 6 , and the a priori transit probability for HD $7924 \mathrm{~b}$ is 0.06 , follow-up campaigns are warranted given the extraordinary value of a nearby transiting super-Earth. Further, while the radius of HD $7924 \mathrm{~b}$ is unknown, we can place bounds of $R_{\mathrm{pl}}=1.4-6 R_{\oplus}$ for planet models spanning pure iron to pure hydrogen (Seager et al. 2007). These extremes produce transits of depth 0.3 and $6 \mathrm{mmag}$, respectively. A more plausible silicatewater planet with $R_{\mathrm{pl}} \approx 2 R_{\oplus}$ (Seager et al. 2007) produces a $\sim 0.6$ mmag transit, near our detection limit.

With an astrometric signature of $0.5 \mu$ as, detecting HD 7924b astrometrically will only be possible with NASA's Space Interferometry Mission (SIM) or its proposed variants (e.g. Sim-Lite and Planet Hunter) that achieve a single measurement precision of $\lesssim 1 \mu$ as. Additional planet(s) of comparable mass in larger orbits, should they exist, would presumably be easier to detect.

Direct imaging of HD 7924b is infeasible for the foreseeable future given the maximum projected separation of $\sim 15$ mas for this short-period planet.

As Doppler velocity surveys probe the observationally challenging realm of super-Earths and Neptune-mass planets, robust detections require continued improvement in velocity precision, an increased number of observations per target, and extra care interpreting the velocities with careful consideration of the null hypothesis. Because the Doppler velocity amplitudes for these lowest mass planets are similar in magnitude to the single measurement precision, we are in an era where it is important to demonstrate the fundamental velocity precision and long-term instrumental stability, and to provide velocity data so that others may check the confidence level of the claim and explicitly consider the null hypothesis by carrying out FAP estimates and other tests. These safeguards will ensure continued confidence and credibility of the profound and extraordinary claims of the detection of low-mass planets.

We gratefully acknowledge the efforts and dedication of the Keck Observatory staff. We are also grateful to the time assignment committees of NASA, NOAO, and the University of California for their generous allocations of observing time. We acknowledge R. Paul Butler and S. S. Vogt for many years 
of contributing to the data presented here. A. W. H. gratefully acknowledges support from a Townes Post-doctoral Fellowship at the U. C. Berkeley Space Sciences Laboratory. J.A.J. is an NSF Astronomy and Astrophysics Postdoctoral Fellow and acknowledges support form NSF grant AST-0702821. G.W.M. acknowledges NASA grant NNX06AH52G. G.W.H. acknowledges support from NASA, NSF, Tennessee State University, and the State of Tennessee through its Centers of Excellence program. Finally, the authors wish to extend special thanks to those of Hawaiian ancestry on whose sacred mountain of Mauna Kea we are privileged to be guests. Without their generous hospitality, the Keck observations presented herein would not have been possible.

\section{REFERENCES}

Alibert, Y., Mordasini, C., Benz, W., \& Winisdoerffer, C. 2005, A\&A, 434, 343 Beaulieu, J.-P., et al. 2006, Nature, 439, 437

Bennett, D. P., et al. 2008, ApJ, 684, 663

Bonfils, X., et al. 2005, A\&A, 443, L15

Butler, R. P., Marcy, G. W., Williams, E., McCarthy, C., Dosanjh, P., \& Vogt, S. S. 1996, PASP, 108, 500

Cumming, A. 2004, MNRAS, 354, 1165

Cumming, A., Butler, R. P., Marcy, G. W., Vogt, S. S., Wright, J. T., \& Fischer, D. A. 2008, PASP, 120, 531

Fischer, D. A., \& Valenti, J. 2005, ApJ, 622, 1102

Forveille, T., et al. 2009, A\&A, 493, 645

Gaidos, E. J., Henry, G. W., \& Henry, S. M. 2000, AJ, 120, 1006

Henry, G. W. 1999, PASP, 111, 845

Ida, S., \& Lin, D. N. C. 2004, ApJ, 604, 388

Ida, S., \& Lin, D. N. C. 2008, ApJ, 673, 487

Jansson, P. 1995, Deconvolution: With Applications in Spectroscopy (New York: Academic)

Johnson, J. A., Butler, R. P., Marcy, G. W., Fischer, D. A., Vogt, S. S., Wright, J. T., \& Peek, K. M. G. 2007, ApJ, 670, 833
Kenyon, S. J., \& Bromley, B. C. 2006, AJ, 131, 1837

Lovis, C., et al. 2006, Nature, 441, 305

Marcy, G. W., \& Butler, R. P. 1992, PASP, 104, 270

Marcy, G., Butler, R. P., Fischer, D., Vogt, S., Wright, J. T., Tinney, C. G., \& Jones, H. R. A. 2005a, Progress of Theoretical Physics Supplement, 158, 24

Marcy, G. W., Butler, R. P., Vogt, S. S., Fischer, D. A., Henry, G. W., Laughlin, G., Wright, J. T., \& Johnson, J. A. 2005b, ApJ, 619, 570

Mayor, M., et al. 2009, A\&A, 493, 639

Mordasini, C., Alibert, Y., Benz, W., \& Naef, D. 2008, in ASP Conf. Ser. 398, Extreme Solar Systems, ed. D. Fischer, F. A. Rasio, S. E. Thorsett, \& A. Wolszczan (San Francisco, CA: ASP), 235

Naef, D., et al. 2001, A\&A, 375, L27

O’Toole, S. J., Tinney, C. G., Jones, H. R. A., Butler, R. P., Marcy, G. W., Carter, B., \& Bailey, J. 2009, MNRAS, 392, 641

Paulson, D. B., Saar, S. H., Cochran, W. D., \& Henry, G. W. 2004, AJ, 127, 1644

Perryman, M. A. C., et al. 1997, A\&A, 323, L49

Rivera, E. J., et al. 2005, ApJ, 634, 625

Saar, S. H., Butler, R. P., \& Marcy, G. W. 1998, ApJ, 498, L153+

Seager, S., Kuchner, M., Hier-Majumder, C. A., \& Militzer, B. 2007, ApJ, 669, 1279

Takeda, G., Ford, E. B., Sills, A., Rasio, F. A., Fischer, D. A., \& Valenti, J. A. 2007, ApJS, 168, 297

Udry, S., Mayor, M., \& Santos, N. C. 2003, A\&A, 407, 369

Udry, S., et al. 2007, A\&A, 469, L43

Valenti, J. A., Butler, R. P., \& Marcy, G. W. 1995, PASP, 107, 966

Valenti, J. A., \& Fischer, D. A. 2005, ApJS, 159, 141

van Leeuwen, F. 2007, A\&A, 474, 653

Vogt, S. S., et al. 1994, in Proc. SPIE Instrumentation in Astronomy VIII, Vol. 2198, ed. David L. Crawford \& Eric R. Craine (Bellingham, WA: SPIE), 362

Wolszczan, A., \& Frail, D. A. 1992, Nature, 355, 145

Wright, J. T. 2005, PASP, 117, 657

Wright, J. T., \& Howard, A. W. 2009, ApJS, in press

Wright, J. T., Marcy, G. W., Butler, R. P., \& Vogt, S. S. 2004, ApJS, 152, 261

Wright, J. T., Upadhyay, S., Marcy, G. W., Fischer, D. A., Ford, E. B., \& Johnson, J. A. 2009, ApJ, 693, 1084 